Article

\title{
Development of a Microfluidic Platform for Trace Lipid Analysis
}

\author{
Andrew Davic ${ }^{1}$ and Michael Cascio ${ }^{1, *}$ \\ 1 Department of Chemistry and Biochemistry, Duquesne Univeristy \\ * Correspondence: casciom@duq.edu
}

\begin{abstract}
The inherent trace quantity of primary fatty acid amides found in biological systems presents challenges for analytical analysis and quantitation, requiring a highly sensitive detection system. The use of microfluidics provides a green sample preparation and analysis technique through small-volume fluidic flow through micron-sized channels embedded in a PDMS device. Microfluidics provides the potential of having a micro total analysis system where chromatographic separation, fluorescent tagging reactions, and detection are accomplished with no added sample handling. This study describes the development and optimization of a microfluidic-laser indued fluorescence (LIF) analysis and detection system that can be used for the detection of ultra-trace levels of fluorescently tagged primary fatty acid amines. A PDMS microfluidic device was designed and fabricated to incorporate droplet-based flow. Droplet microfluidics have enabled on-chip fluorescent tagging reactions to be performed quickly and efficiently, with no additional sample handling. An optimized LIF optical detection system provided fluorescently tagged primary fatty acid amine detection sub-fmol ( $436 \mathrm{amol})$ LODs. The use of this LIF detection provides unparalleled sensitivity, with detection limits several orders of magnitude lower than currently employed LC-MS techniques and might be easily adapted for use as a complementary quantification platform for parallel MS-based -omics studies.
\end{abstract}

Keywords: primary fatty acid amides; microfluidics; laser induced fluorescence; bioactive lipids

\section{Introduction}

Primary fatty acid amides (PFAMs) are a subclass of fatty acyls important in nervous system signaling, receptor binding, and numerous other physiological roles [1]. Structurally, these bioactive lipids contain a carboxamide head group and an acyl tail varying in both length and unsaturation. Palmitamide (C16:0), palmitoleamide (C16:19), oleamide (C18:19-cis), elaidamide (C18:19-trans), and linoleamide (C18:29,12) were the first PFAMs to be identified in 1989 from luteal phase plasma [2]. Oleamide is widely studied due to its abundance of physiological roles and has been found (along with erucamide [C22:13] ) in the cerebrospinal fluid of sleep deprived cats [3-5]. In addition to sleep induction, systematic administration of oleamide effects vasorelaxation, decreased locomotor activity, and decreased body temperature [6-8]. Although not classified as an endocannabinoid, oleamide displays characteristic properties such as analgesia, increased appetite, and antianxiety upon high concentration binding to the cannabinoid receptors $[9,10]$. Oleamide has been shown to be highly selective in potentiating the action of serotonin on serotonin receptors (5- $\mathrm{HT}_{2 \mathrm{~A}}$ and $5-\mathrm{HT}_{2 \mathrm{C}}$ ), which produces long-lasting inhibition of $\mathrm{GABA}_{\mathrm{A}}$ receptor function, and thereby effects alertness, sleep, and mood [11-14]. It has also proven to be effective at blocking gap junction communication between rat glial cells, while not effecting intercellular calcium signaling $[15,16]$. Other highly-characterized PFAMs include erucamide, which modulates water balance 
and stimulates angiogenesis [17-19], linoleamide, which induces increases in $\mathrm{Ca}^{2+}$ concentrations in Madin-Darby Canine Kidney (MDCK) tubular cells, aiding in the initiation and regulation of many cell functions [20-22], and anandamide (C20:45,8,11,14), which acts as a endogenous ligand for cannabinoid receptors [23-25].

The vast array of physiological properties exhibited by PFAMs makes their study attractive in the realm of disease-state biomarker research, however, their endogenous low (nM) concentrations present analysis and detection challenges. PFAMs have been found in plasma, cerebral spinal fluid, neuroblastoma cells, and neuronal tissue $[2,3,23,26]$. Initial discovery of PFAMs utilized nuclear magnetic resonance spectroscopy; however, current work more commonly involves coupling gas chromatography (GC) or high performance liquid chromatography (HPLC) for separation with mass spectrometry (MS) for sensitive detection and structural identification of endogenous or derivatized analyte [2-5, 17, 23, 24, 26-33]. MS detection, although widely employed and of great benefit, results in limits of detection and quantitation on the periphery of endogenous PFAM concentrations, thus a complementary methodology for sensitive detection is required. The potential sensitivity of laser induced fluorescence (LIF) makes this method an attractive alternative detection system.

The use of fluorescent labeling coupled with LIF detection presents the theoretical ability to approach single molecule detection using a fluorophore of high quantum yield versus the relatively moderate ionization efficiency which can be a limiting factor in MS-based detection systems. In addition, LIF detection can also provide information regarding relative concentrations of comparative chemical species, a benefit not afforded by MS which only allows direct comparison between isotopically labeled chemical isomers that ionize equally. In the case of PFAMs, the amide must first be converted to its conjugate amine as the carbonyl of the amide causes delocalization of electron density, hindering the fluorescent reaction [34]. To achieve maximal fluorescent labeling efficiency, a microfluidic platform can be utilized. Microfluidics is the general label given to the technology of systems that process or manipulate small volumes $\left(10^{-9}\right.$ to $\left.10^{-18} \mathrm{~L}\right)$ of fluids by utilizing embedded channels with internal diameters on the micrometer scale [35]. Microchip fabrication can be performed quickly, safely, and economically via soft lithography using compounds such as polydimethylsiloxane (PDMS)[36]. An inherent obstacle faced while performing microfluidic reactions on-chip is mixing efficiency, as laminar flow dominates the system at the micro-scale due to the low Reynolds number $(\operatorname{Re}<2300)$. In laminar flow, the major form of mixing that occurs is diffusion between multiple streamlines, which is highly ineffective. Segmented flow microfluidics presents the ability to conduct highly efficient reactions on-chip entirely within small-volume $(\mathrm{nL})$ microdroplets. This may be performed by introducing at least two immiscible phases into the micro-channels via pressure-driven flow. One phase consists of a carrier fluid (perfluorodecalin, gas, etc.), which is continuous and encapsulates the second phase (analyte and all reaction-dependent solutions) into segmented nanodroplets [37]. Highly efficient mixing of nanodroplets relies on the repeated stretching and folding of the intra-droplet fluidic layers until these layers become increasingly thin to the point where inter-layer diffusion becomes rapid [38, 39]. The mixing process, known as chaotic advection, drastically reduces reaction time while improving reaction efficiency largely due to the significantly reduced mean free path as well as the absence of diffusion outside of the nanodroplet [40]. As the droplets flow through the microchannels, frictional forces introduced via contact with the channel walls causes internal recirculation of the 
droplet, enhancing mixing. Implementing winding "S" shaped channels with several turns also enhances mixing by reorienting the droplet's internal fluidic layers in the direction of droplet movement [40-42].

Herein, we describe our efforts to develop the methodologies of droplet-based microfluidics to enhance the fluorescent labeling of derivatized PFAMs. The use of $\mu$ HPLC with single photon counting detection will enable an in-line micro total analysis system ( $\mu \mathrm{TAS})$ with detection limits sufficient for investigation of endogenous PFAMs. Significantly, this approach provides a sensitive, quantifiable method more sensitive than typical MS-based studies, so it can provide a complementary isotope-independent adjunct to discovery-based MS studies.

\section{Results}

2.1. Fluorescent Tagging of Primary Amines. Ultra-trace detection of primary amines has been achieved by derivatization with fluorescent reagents. An attractive flourogen of choice is NDA, due to commercial availability, inexpensive cost, and simple reaction schema. At equimolar concentrations, varying the reactant (primary amine, $\mathrm{KCN}, \mathrm{NDA}$ ) ratios directly affects the fluorescence response intensity. In examining reaction stoichiometries, the optimal reaction conditions of those tested in vitro at approximately $45^{\circ} \mathrm{C}$ for 30 minutes was 1:20:24 (amine:KCN:NDA) (Figure 1). Given potential red and blue-shifting as a function of solvent, the emission wavelength was optimized for our solvent conditions to maximize $\mathrm{S} / \mathrm{N}$ as a function of emission wavelength. Using an excitation laser at $405 \mathrm{~nm}$, the experimentally determined emission maxima (Emmax) is approximately $470 \mathrm{~nm}$ (Figure 2). This Stokes shift of approximately $65 \mathrm{~nm}$ is wide enough to ensure that no reflected excitation radiation will affect the background signal. To ensure proper fluorescent tagging of the primary amine and determine any native background fluorescence, all individual reactants and reaction combinations (maintaining 1:20:24 molar ratio, with methanol substituted for reactants not used) were examined using HPLC with fluorescence detection, as shown in Figure 3. An isocratic elution using $100 \%$ methanol and a reversed phase $\mathrm{C}_{18}$ column were used. In control studies, none of the individual reactants display any fluorescence response (Figure $3 \mathrm{~A}-\mathrm{C}$ ), nor do binary mixtures of the amine-NDA and amine-KCN reactions (Figure 3D and E, respectively). It should be noted that although amine-NDA and amine-KCN show no fluorescence response, the KCN-NDA binary mixture shows a relatively early eluting fluorescence peak, likely caused by dimerization of the NDA upon reaction with KCN. In the full reaction using a 12 and 10 carbon amine, the KCN-NDA dimerization peak is visible at 1.5 minutes, at a much lower response as compared with the tagged amines (Figure $3 \mathrm{G}$ and $\mathrm{H}$, respectively). A chromatogram of four fully saturated primary amines of acyl chain length varying from 10 to 16 carbons is shown in Figure 3I. Although baseline resolution could not be achieved between C10:0 and C12:0, the resolution between consecutive saturated amines does increase in accordance with increasing acyl chain length, with baseline resolution between C12:0 and C14:0, and all subsequent amines of increasing acyl chain length. It should be noted that biologically relevant amides typically contain acyl chain lengths of 16 or more carbons with varying degrees of unsaturation. Should co-elution occur between biologically relevant amines, mobile phase polarity can be reduced or the use of a gradient elution profile can easily be employed. 


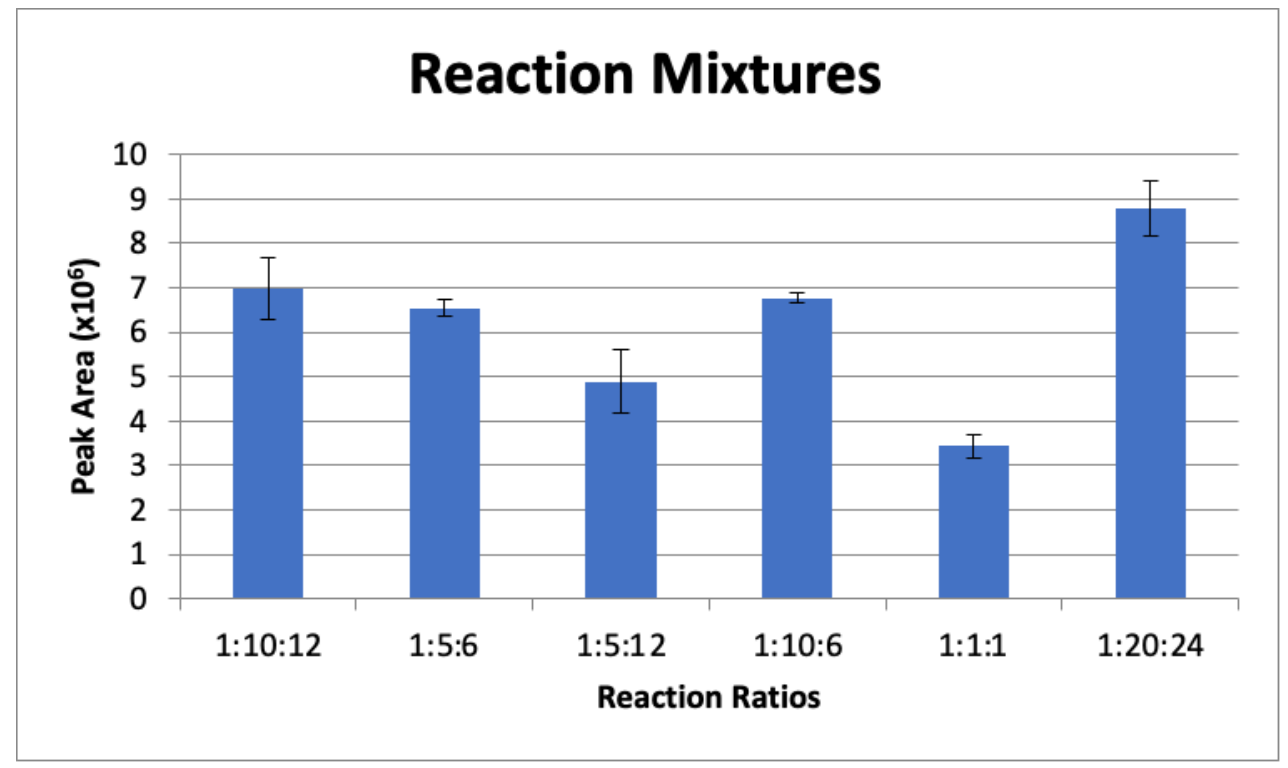

Figure 1. Reaction mixtures as a function of reactants stoichiometry. All solutions were prepared with reactants added in equimolar concentrations following the format of decylamine:KCN:NDA by volume. Average peak area of fluorescently tagged decylamine diluted to a concentration of $1 \mu \mathrm{M}$ is shown for each reaction mixture, with standard deviations depicted $(n=3)$.

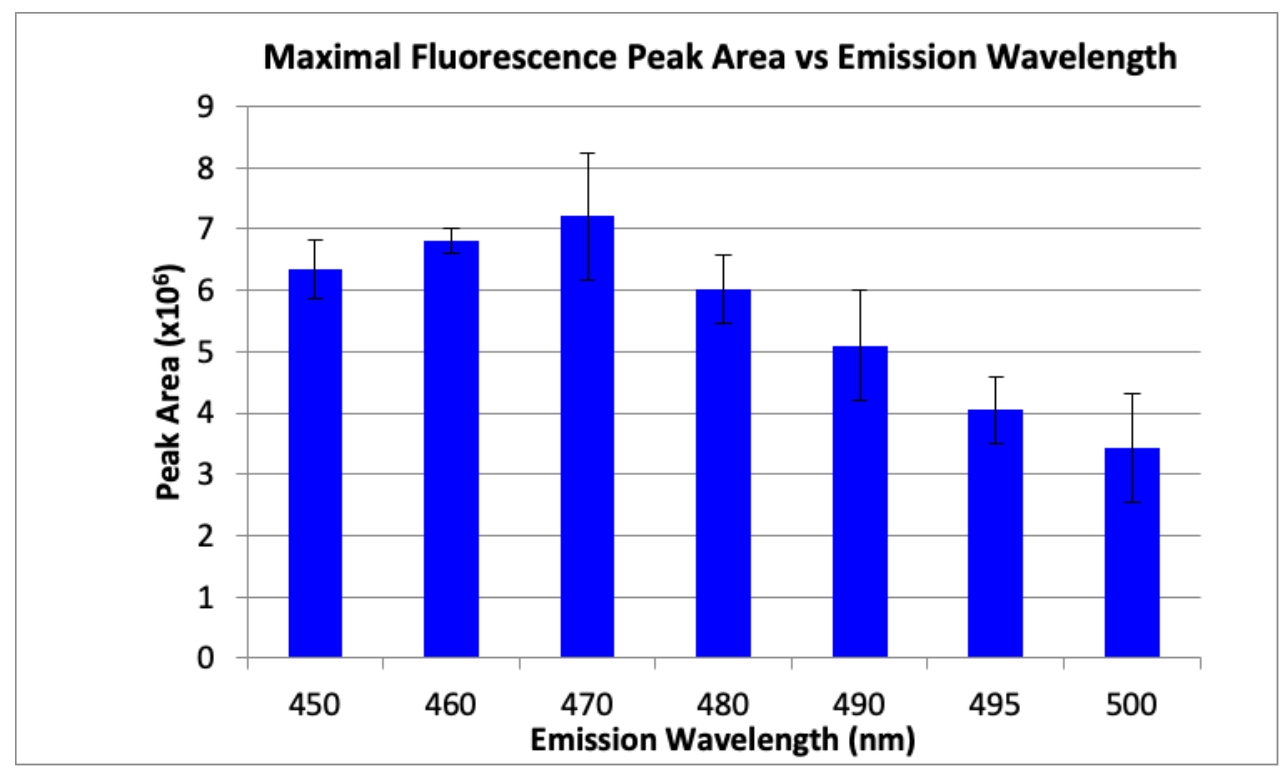

Figure 2. Average peak areas are shown at varying intervals of fluorescence emission wavelength. Dodecylamine was fluorescently tagged with NDA and diluted to a concentration of $1 \mu \mathrm{M}$. Fluorescence emission wavelengths ranged from 450 to $500 \mathrm{~nm}$ with the excitation wavelength held at $405 \mathrm{~nm}$. Standard deviations are shown $(\mathrm{n}=3)$. 

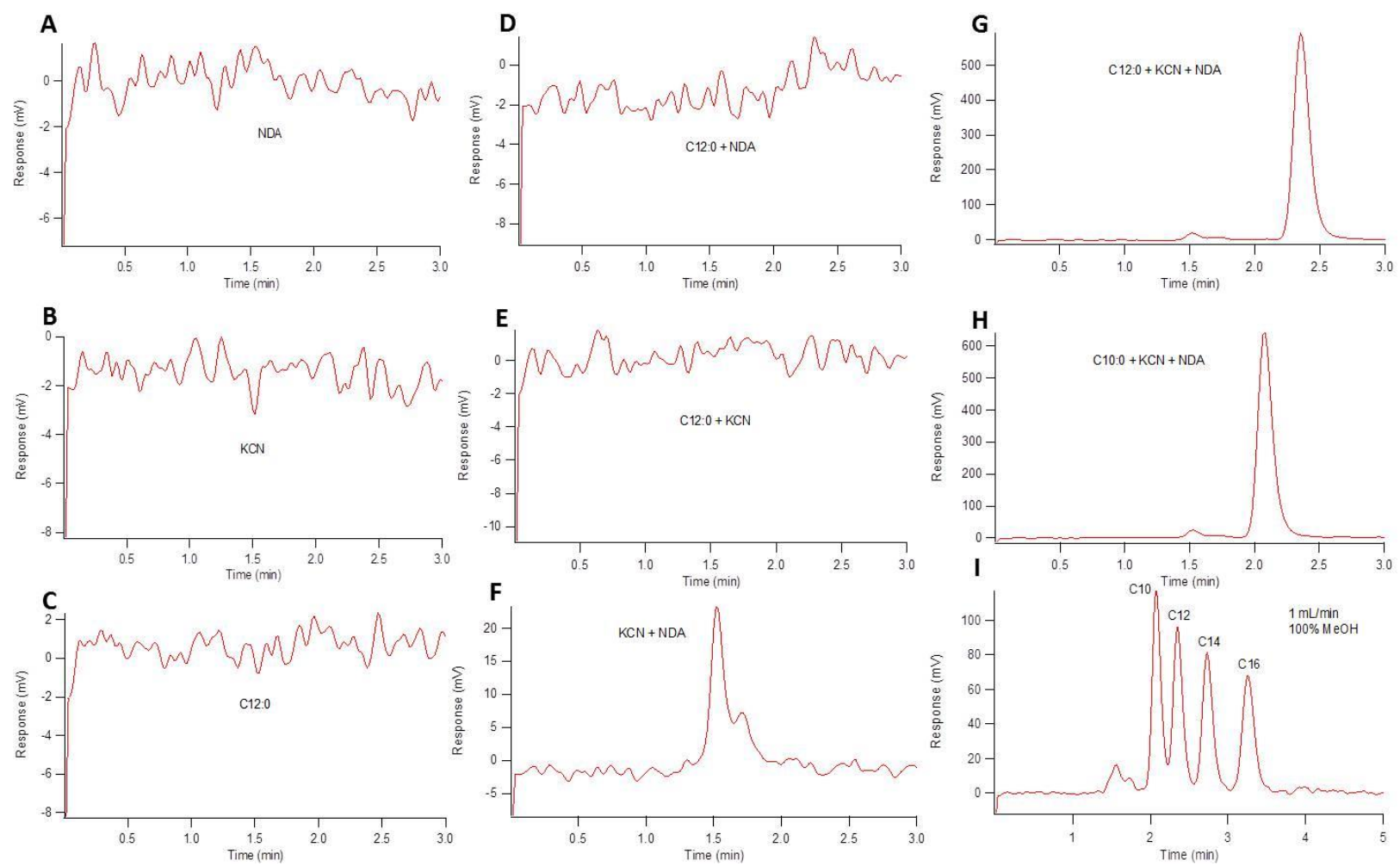

Figure 3. HPLC chromatograms showing native fluorescence of reactants and varying reactant mixtures. All reactants were prepared to $100 \mu \mathrm{M}$ and incubated at $50^{\circ} \mathrm{C}$ for 45 minutes. For mixtures, 1:10:12 (v/v/v) equimolar amine:KCN:NDA reaction conditions were used. $A . B$. and $C$. show fluorescence chromatograms of NDA, $\mathrm{KCN}$, and dodecylamine, respectively. D. and E. show dodecylamine reacted with NDA and dodecylamine reacted with $K C N$, respectively. $F$. shows the fluorescence response of KCN and NDA reacted with one another. G. and H. show the full reaction of NDA-tagged dodecylamine and decylamine, respectively, for perspective with respect to retention time. I. NDA-tagged amines (decylamine, dodecylamine, tetradecylamine, and octadecylamine). All elution profiles were isocratic with flow rates of $1 \mathrm{~mL} / \mathrm{min}$.

2.2. Segmented Flow Microfluidics. Droplet-based, or segmented flow, microfluidics is an advantageous technique to use in that it allows for highly efficient reactions, approximately 100x less solvent usage as compared to HPLC, and compatibility with highly sensitive photon detectors. The process of droplet formation has been reviewed and illustrated especially well by Song et al.[40]. The microchannel features were designed for specific application to fluorescent tagging of polar neutral lipids and is shown in detail in Figure 4. Figure 4A shows a graphical representation of the microchannel features with four inlet ports along the bottom of the chip and one outlet port at the top of the chip. The far left inlet flows an immiscible oil phase, perfluorodecalin, while the other three are designed for the reactants. The far right inlet has been elongated and includes a fritless weir near the junction point to allow for potential future chromatographic packing on-chip. All of the flow is pressure driven via external syringe pumps, with the immiscible phase flowing at a slightly higher flow rate than the sum of the reactants (all reactants' flow rate is equal coming from a multi-port syringe pump). This difference in flow creates a droplet-producing taper effect where the reactants' flow combines immediately prior to the junction and the resulting droplets are carried through the channel by the immiscible phase. Due to the laminar nature of the flow through a straight channel, the droplets contain distinct layers representative of each reactant, with inefficient mixing occurring via simple diffusion between each intra-droplet fluidic layer. As the droplet passes through the curved region of the channel, it undergoes stretching, folding, and reorientation due to the sheer forces applied by the channel walls. Each turn in the channel doubles the fluidic layers, to the point where they become increasingly thin, causing inter-layer diffusion to be an incredibly efficient form of mixing. The incident laser is focused centrally on the post-mixing 
region of the channel to fluoresce the sample after complete reaction has occurred. The completeness of the reaction was evaluated by collecting a fluorescence chronogram of droplet fluorescence from equimolar reactants flowing at identical rates. A previous channel design that incorporated only 10 turns in the mixing region resulted in inconsistent droplet fluorescence response (as discussed below and shown in Figure 11). Of note is both the inter- and intra-droplet variation in fluorescence response, indicative of incomplete and inconsistent reactions. The currently used channel design features 140 turns in the mixing region, and the corresponding fluorescence chronogram is shown in Figure 4A and 10. The fluorescence response of each droplet is very consistent and reproducible, indicating a completed reaction on-chip.

A

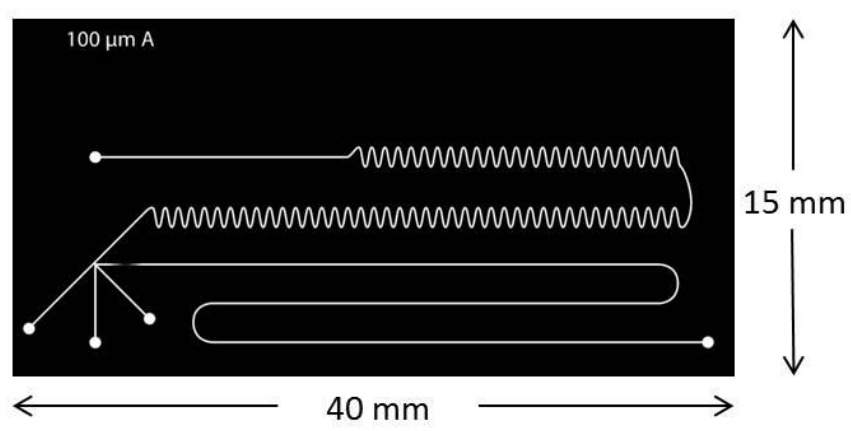

B

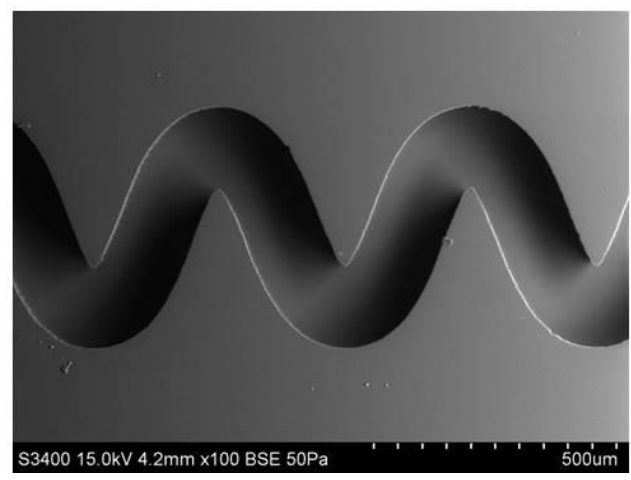

$\mathrm{C}$

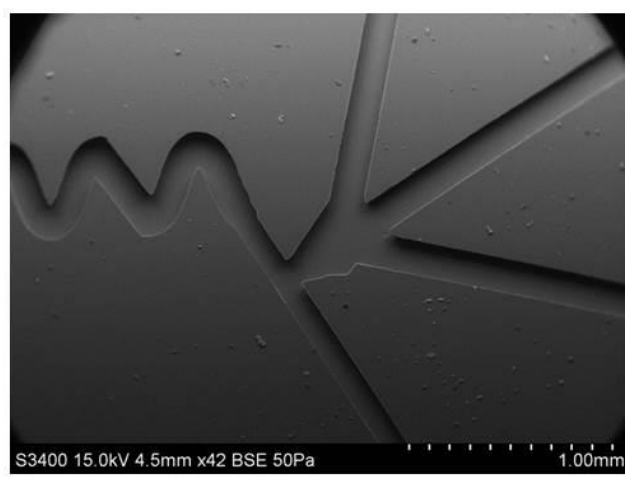

D

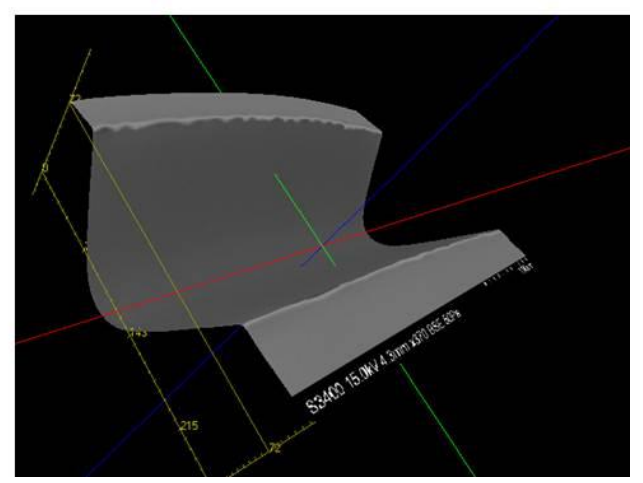

Figure 4. SEM imaging of channel features of a microfluidic device fabricated using SU-8 2075 photoresist. (A) Cartoon schematic of final photomask used for PDMS microfluidic chip fabrication. $(B)$ The four inlet channels, T-junction, and beginning of the mixing region is shown in a zoomed out image. (C) A zoomed in image of the mixing channel shows consistent curving features which allow for on-chip mixing and reactions $(D)$ The three dimensional image can be used to calculate distances between two points, enabling the determination of channel width and depth.

2.3. LIF Optical Detection System. All optical components used in the LIF detection system must be meticulously arranged and aligned to ensure maximum signal to noise ratio (S/N). A representative schematic of the optical detection system is shown in Figure 5. The incident laser beam is produced from a vertically polarized diode-pumped solid state crystal laser at a wavelength of $405 \mathrm{~nm}$. The beam is reflected off of a series of mirrors and passes through a $405 \mathrm{~nm}$ bandpass excitation filter. A pinhole filter removes the low-intensity laser scatter that occurs at the outer circumference of the beam. Our initial optical system utilized a rotation-mounted linear polarizer set to $300^{\circ}$, as this was expected to elicit maximum signal. However, further studies revealed a $S / N$ increase of approximately 13\% upon removing the polarizer altogether (unpublished observation). A longpass dichroic mirror with a cutoff wavelength of $425 \mathrm{~nm}$ was used to reflect incident excitation wavelength while allowing transmittance of red-shifted emission wavelength. A 40x 
microscopic objective lens is used to focus the laser beam onto the microchannel to excite the NDA-tagged amine sample. The resulting fluorescence emission at $472 \mathrm{~nm}$ passes back through the microscope objective and is transmitted through the dichroic lens. A final mirror reflects the fluorescence through a second pinhole filter and into dark optics tube, which contains a $472 \mathrm{~nm}$ emission bandpass filter as well as achromatic focusing lenses. The fluorescence emission beam is then focused onto the $180 \mu \mathrm{m}$ diameter circular active area of a single photon counting module equipped with a silicon avalanche photodiode. Individual photons generate TTL pulses, which are binned over $25 \mathrm{~ms}$ increments and transmitted to data acquisition software.
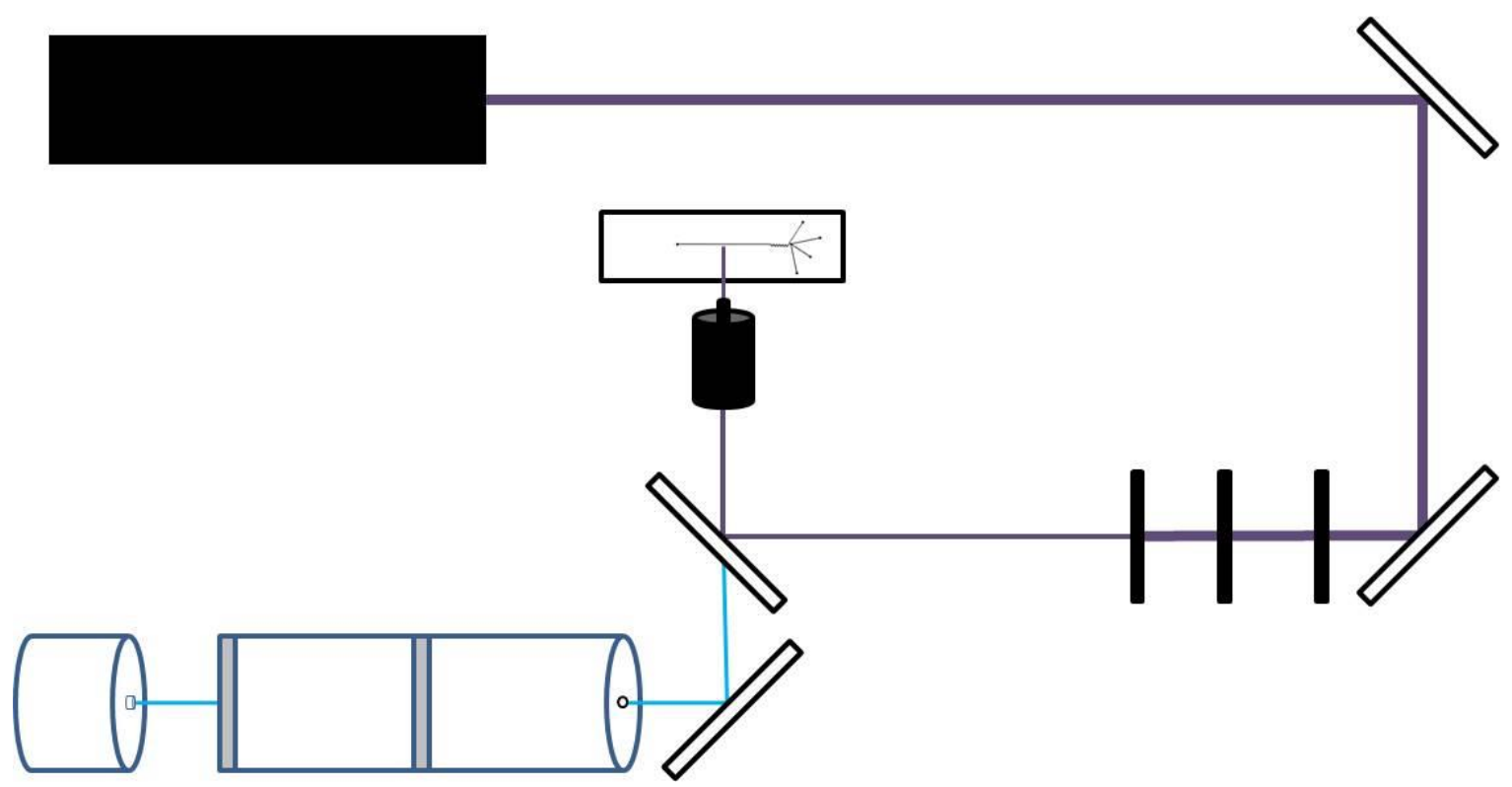

Figure 5. Schematic representation of the LIF detection system. A $405 \mathrm{~nm}$ excitation laser beam is emitted from a solid-state laser $(A)$ and reflected off of two mirrors $(B, C)$. The beam then passes through a $405 \mathrm{~nm}$ emission bandpass filter $(D)$, rotationally-mounted polarizer $(E)$, and pinhole filter $(F)$. The beam reflected off of a $425 \mathrm{~nm}$ dichroic lens $(G)$ and focused onto the sample $(I)$ using a microscope objective $(H)$. The resonant fluorescence beam then passes back through the objective and dichroic lens, is reflected off of another mirror $(J)$, and into the optics tube $(K)$ through a pinhole filter $(L)$. Inside of the tube is a $472 \mathrm{~nm}$ emission bandpass filter $(M)$ and achromatic focusing lenses $(N)$. The focused beam is detected by a single photon counting APD $(O)$.

To ensure maximum sensitivity, several components of the optical detection system must be optimized. The variable power source allows the user to control lasing power from 0-50 $\mathrm{mW}$. Using NDA-tagged dodecylamine diluted to $1 \mathrm{uM}$, fluorescence chronograms were acquired using varying lasing power (Figure 6). As the lasing power is increased, both the response and the noise increase accordingly. By plotting the signal to noise ratio $(\mathrm{S} / \mathrm{N})$ versus each respective lasing power, the optimal power setting was found to be $12.7 \mathrm{~mW}$. The laser emits an incident beam that approximates a Gaussian profile, with the center of the beam emitting the highest intensity radiation. As the distance from the center of the beam increases outward, the intensity is reduced, and variability increased. As shown in Figure 7, the response increases as the pinhole diameter increases. Once the approximate maximum response is reached, increasing the pinhole diameter will only increase the noise. Using the maximum emission (Emmax) wavelength of approximately $470 \mathrm{~nm}$, several bandpass (BP) and longpass (LP) emission filters were tested using an NDA-tagged primary amine prepared similarly to the aforementioned. The calculated $\mathrm{S} / \mathrm{N}$ from the corresponding fluorescence chronograms was determined for each filter and is shown in Figure 8. As expected, the $472 \pm 15 \mathrm{~nm}$ BP filter presented the greatest $\mathrm{S} / \mathrm{N}$ as its maximum transmittance $\mathrm{T}_{\max }$ is nearest to the Emmax. Although the $450 \mathrm{~nm}$ LP filter recorded nearly three times the fluorescence response than the $472 \mathrm{BP}$ filter, the greatly widened transmission band with the LP filters adds more than four times the noise, resulting in a less sensitive optical detection system at ultra-trace 
concentration levels. Although both the $470 \mathrm{~nm}$ LP and the $495 \pm 20 \mathrm{~nm}$ BP filters have transmission bands that extend to or near the calculated Em max, the percent transmittance at the Emmax is much lower than the $\mathrm{T}_{\max }$, resulting in a lower $\mathrm{S} / \mathrm{N}$.

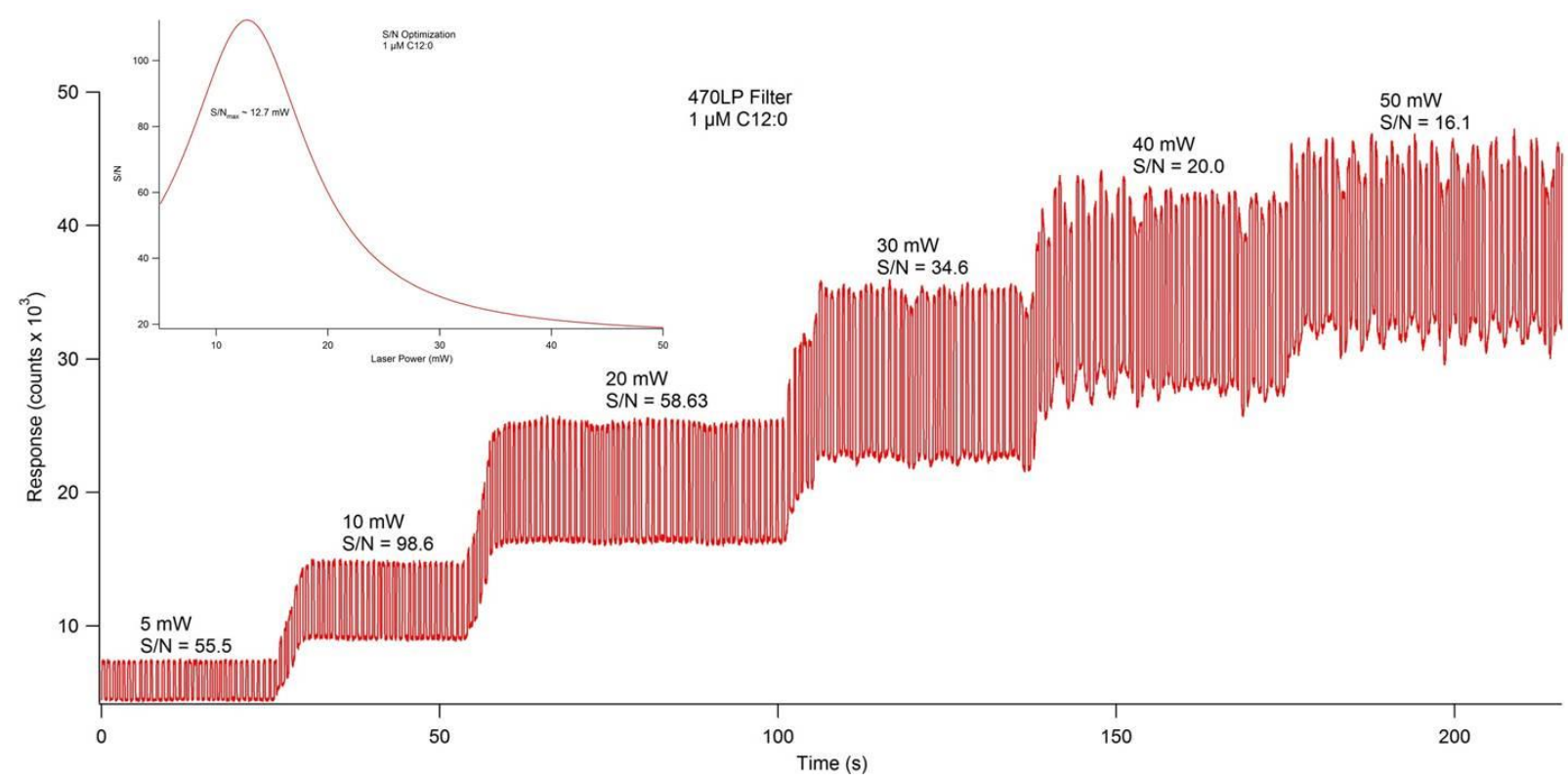

Figure 6. Fluorescence chronogram showing the effect on response and $\mathrm{S} / \mathrm{N}$ caused by lasing power. Using the variable power controller, the lasing power was set to 5, 10, 20,30, 40, and $50 \mathrm{~mW}$ and fluorescence response was recorded at each increment. Of the individual power settings, $10 \mathrm{~mW}$ showed the highest $\mathrm{S} / \mathrm{N}$ at 98.6, but extrapolation of the data fit to a Gaussian distribution showed the $\mathrm{S} / \mathrm{N}_{\mathrm{mx}}$ to be at $12.7 \mathrm{~mW}$ (inset).

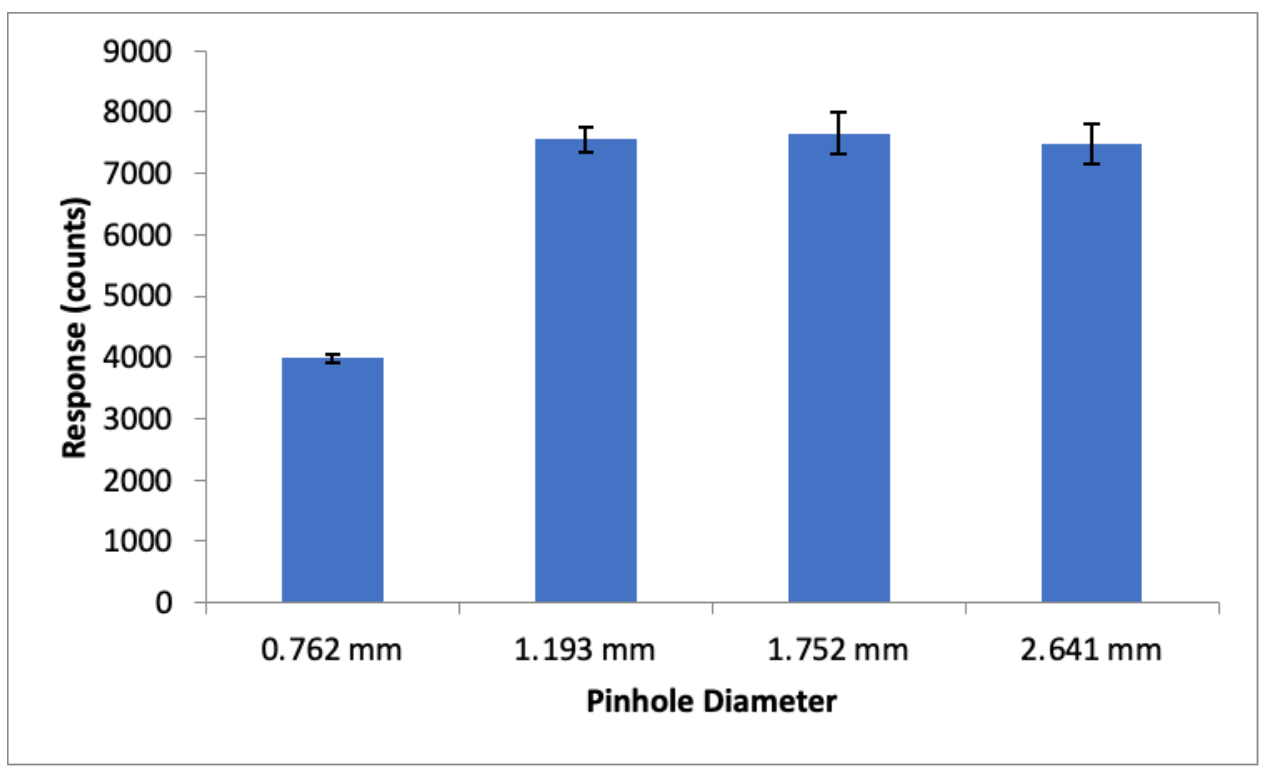

Figure 7. Average fluorescence response shown at each pinhole diameter setting. At a diameter of $0.762 \mathrm{~mm}$, the average response is nearly half of that at $1.193 \mathrm{~mm}$. Further opening the pinhole filter past $1.193 \mathrm{~mm}$ does not significantly increase the average response, but, given the increase in the error, appears to increase the droplet-to-droplet variability $(n=7-10)$. 


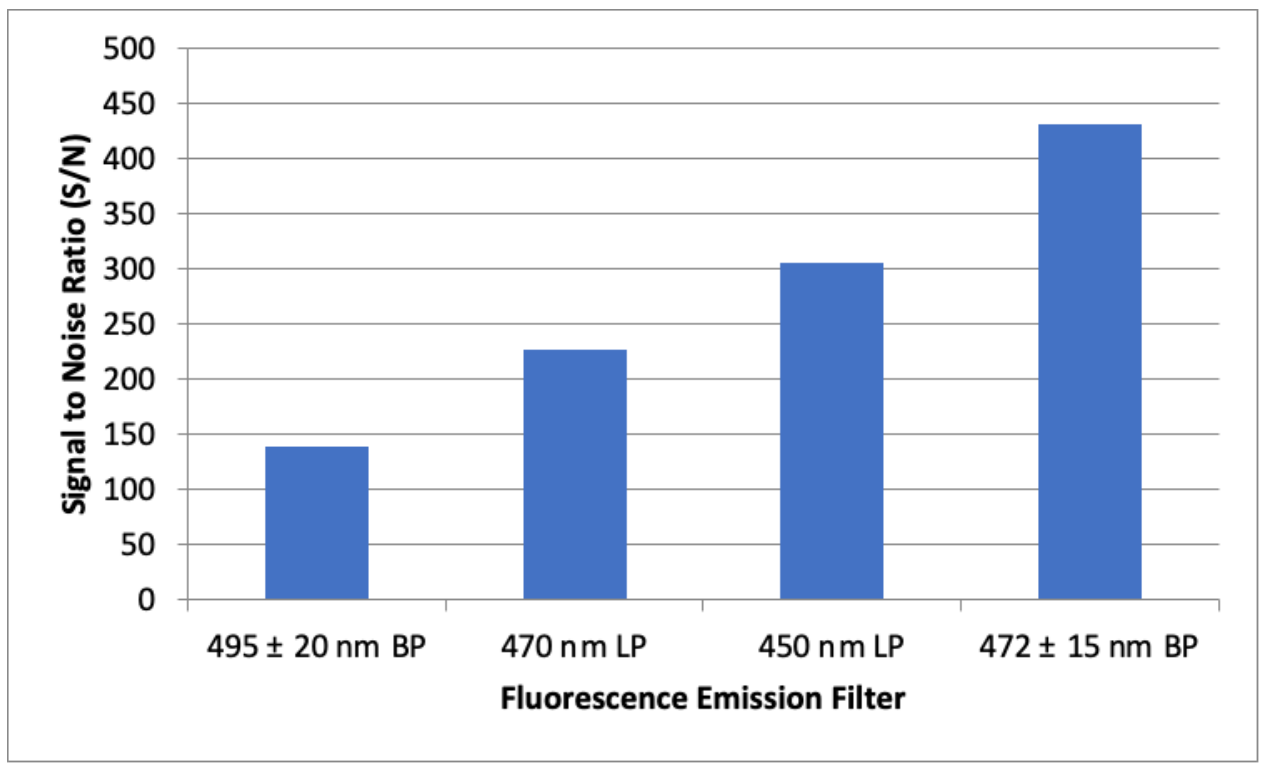

Figure 8. Four different emission filters were tested to determine maximum $\mathrm{S} / \mathrm{N}$ of a $5 \mu \mathrm{M}$ NDA-tagged hexadecylamine solution. The $472 \mathrm{~nm}$ bandpass filter showed the greatest $\mathrm{S} / \mathrm{N}$, with the $450 \mathrm{~nm}$ longpass, $470 \mathrm{~nm}$ longpass, and $495 \mathrm{~nm}$ bandpass filters showing progressive decreasing of the $S / \mathrm{N}$.

2.4. Droplet Formation and On-Chip Fluorescent Tagging. Droplet-based microfluidics presents the ability to conduct highly efficient fluorescent tagging reactions on-chip, minimizing additional sample handling. For optimal rapid and consistent droplet formation, the flow rate of the immiscible oil phase must be greater than that of the reactant phase(s). It was found that a 4 $\mu \mathrm{L} / \mathrm{min}$ flow rate of oil phase to $2 \mu \mathrm{L} / \mathrm{min}$ flow rate of the reactant phase(s) provided the consistent droplet size and frequency (Figure 9). It should be noted that when multiple reactants are used for fluorescent tagging on-chip, the summation of their individual flow rates should equal the $2 \mu \mathrm{L} / \mathrm{min}$ total flow rate. The acquisition software was set to produce a binned data point every $25 \mathrm{~ms}$, which was sufficient to record a true maximum and minimum response for each droplet.

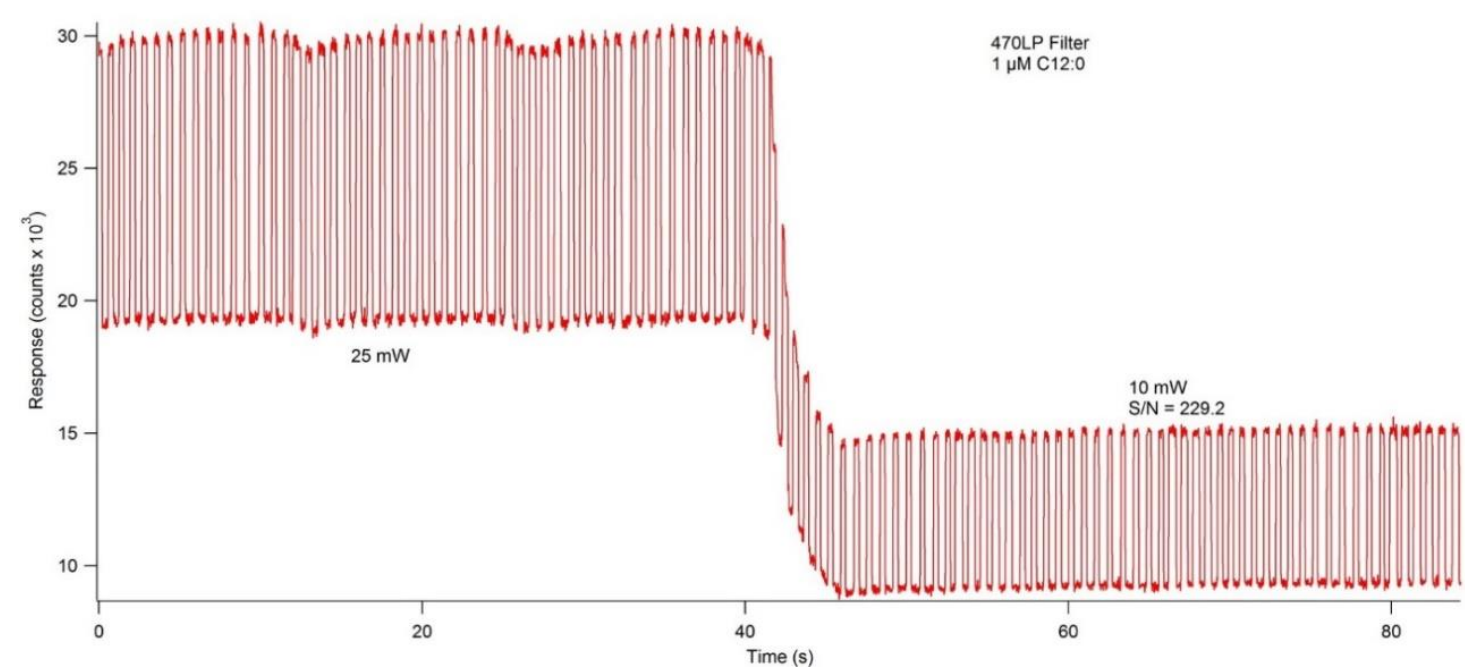

Figure 9. An example of a fluorescence chronogram showing consistent droplet formation. The lasing power is changed mid-acquisition with an immediate adjustment in response. Consistent droplet formation is determined by equal spacing between peaks (frequency) and equal peak widths (size). . 
Detection limit studies were performed using optimized LIF detection system settings, a reactant flow rate of $1.5 \mu \mathrm{L} / \mathrm{min}$, and NDA-tagged dodecylamine serially diluted to an amine concentration of $80.8 \mathrm{nM}$. With a droplet frequency of 4.6 droplets/second, each droplet was calculated to contain $5.4 \mathrm{~nL}$ of solution. At an amine concentration of $80.8 \mathrm{nM}$, the average droplet contained 436 amol of fluorescently tagged amine (Figure 10).

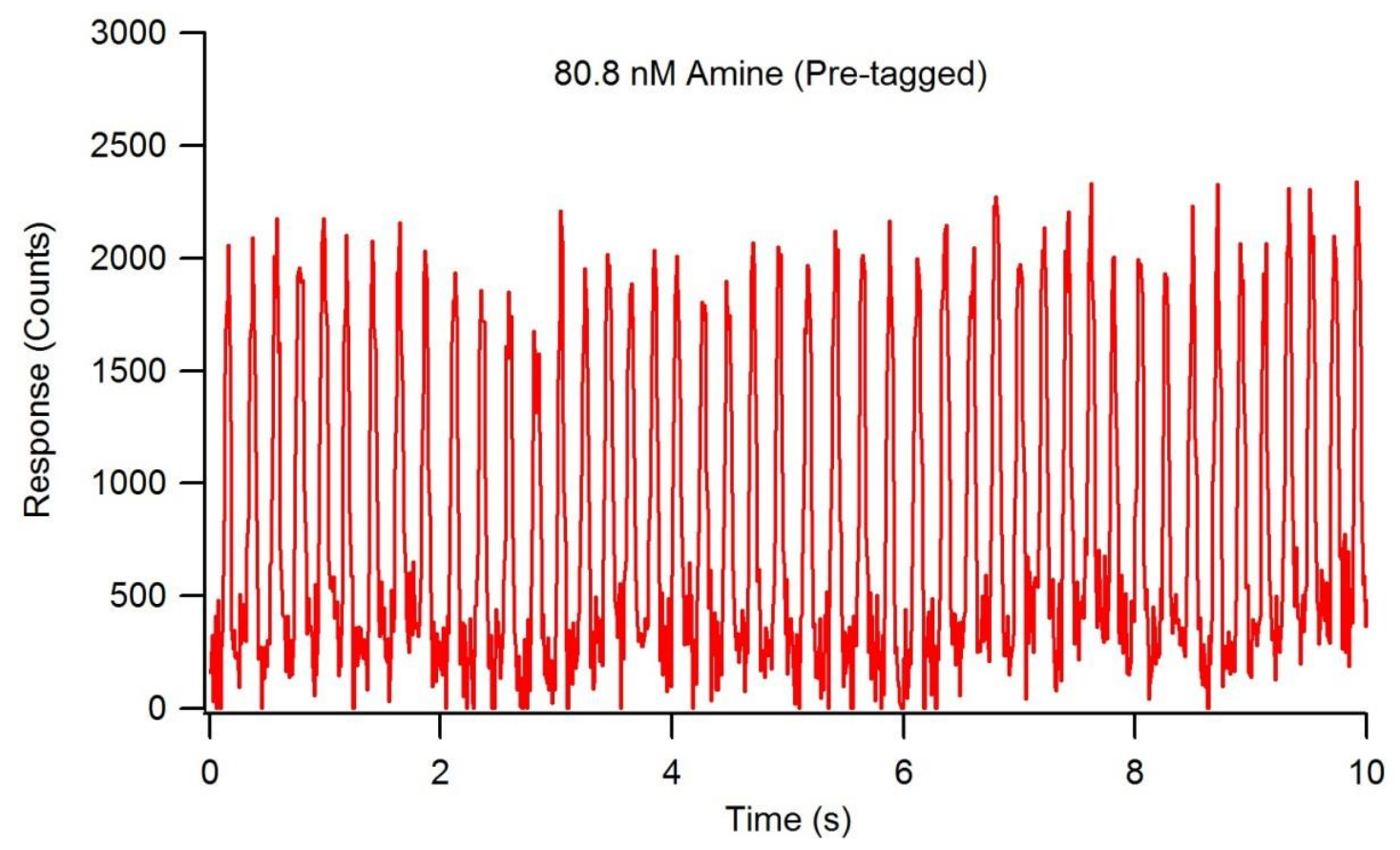

Figure 10. Fluorescence chronogram showing NDA-tagged decylamine approaching the detection limit of the LIF detection system. The tagging reaction occurred pre-chip and the total amine concentration was serially diluted to $80.8 \mathrm{nM}$. The droplet frequency was determined to be 4.6 droplets/second and average droplet volume was $5.4 \mathrm{~nL} /$ droplet, resulting in approximately 436 amol of amine per droplet.

On-chip NDA-tagging efficiency was determined using two different channel designs. A 1:20:24 (M/M/M) amine:KCN:NDA reaction concentration ratio was maintained with all reactants independently administered to the microchip at a flow rate of $0.5 \mu \mathrm{L} / \mathrm{min}(1.5 \mu \mathrm{L} / \mathrm{min}$ total flow $)$. The resulting concentration of hexadecylamine per droplet was calculated to be $3.33 \mu \mathrm{M}$. The initial chip design contained a mixing region with 10 turns in the channel, which resulted in incomplete fluorescent tagging as shown by inconsistencies in maximum droplet response by the fluorescence chronogram (Figure 11). Transitioning to a new chip design containing 130 turns in the mixing region of the channel resulted in a completed fluorescent tagging reaction, as indicated by consistent maximum responses per droplet (Figure 12). 


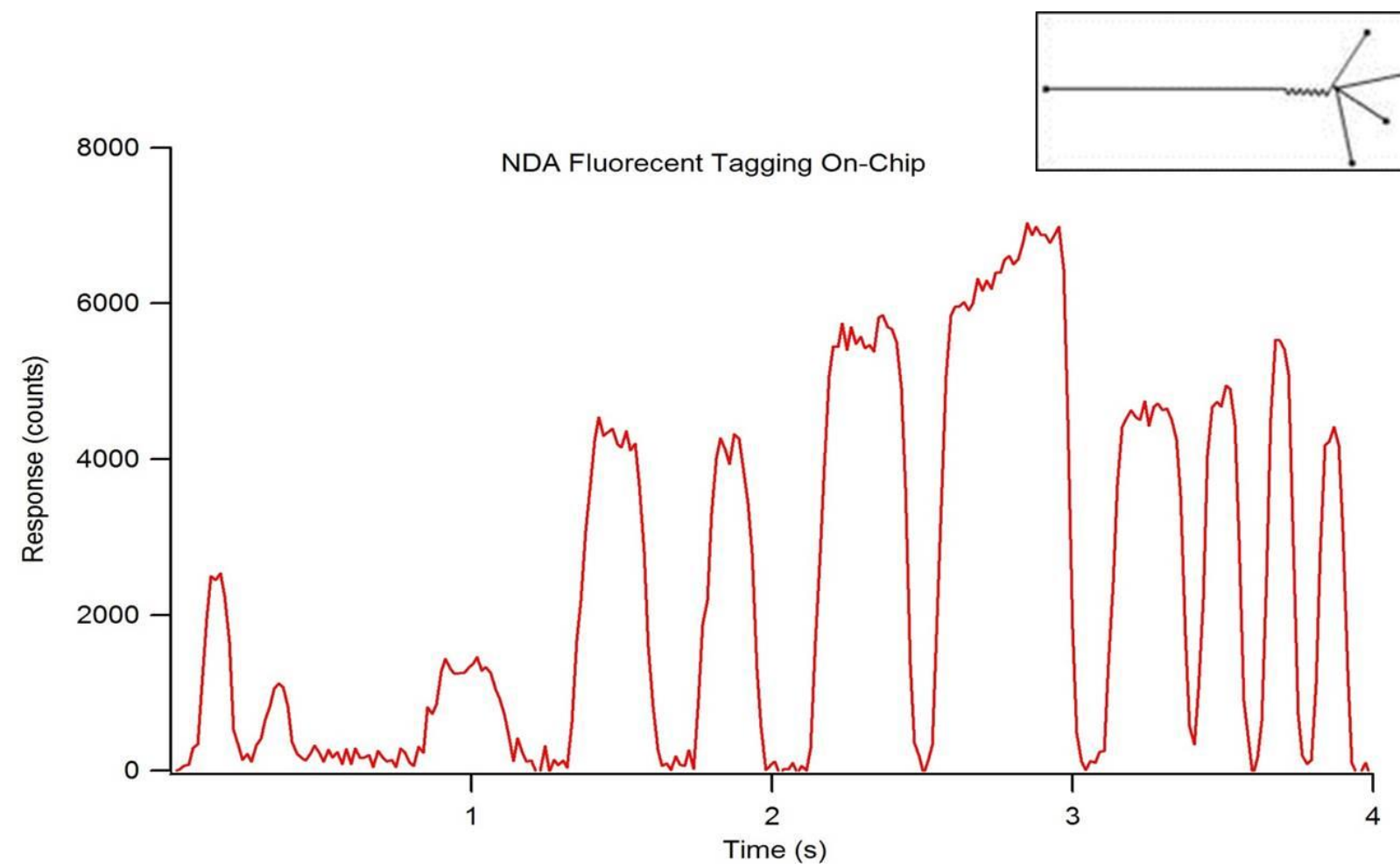

Figure 11. Fluorescence chronogram showing NDA-tagging reaction efficiency performed on-chip. Reactants were maintained in a 1:20:24 amine:KCN:NDA molar ratio and applied to the chip independently at $0.5 \mu \mathrm{L} / \mathrm{min}$. Using a chip design with 10 turns in the mixing region (inset) resulted in an incomplete reaction, indicative of inconsistent maximum peak responses.

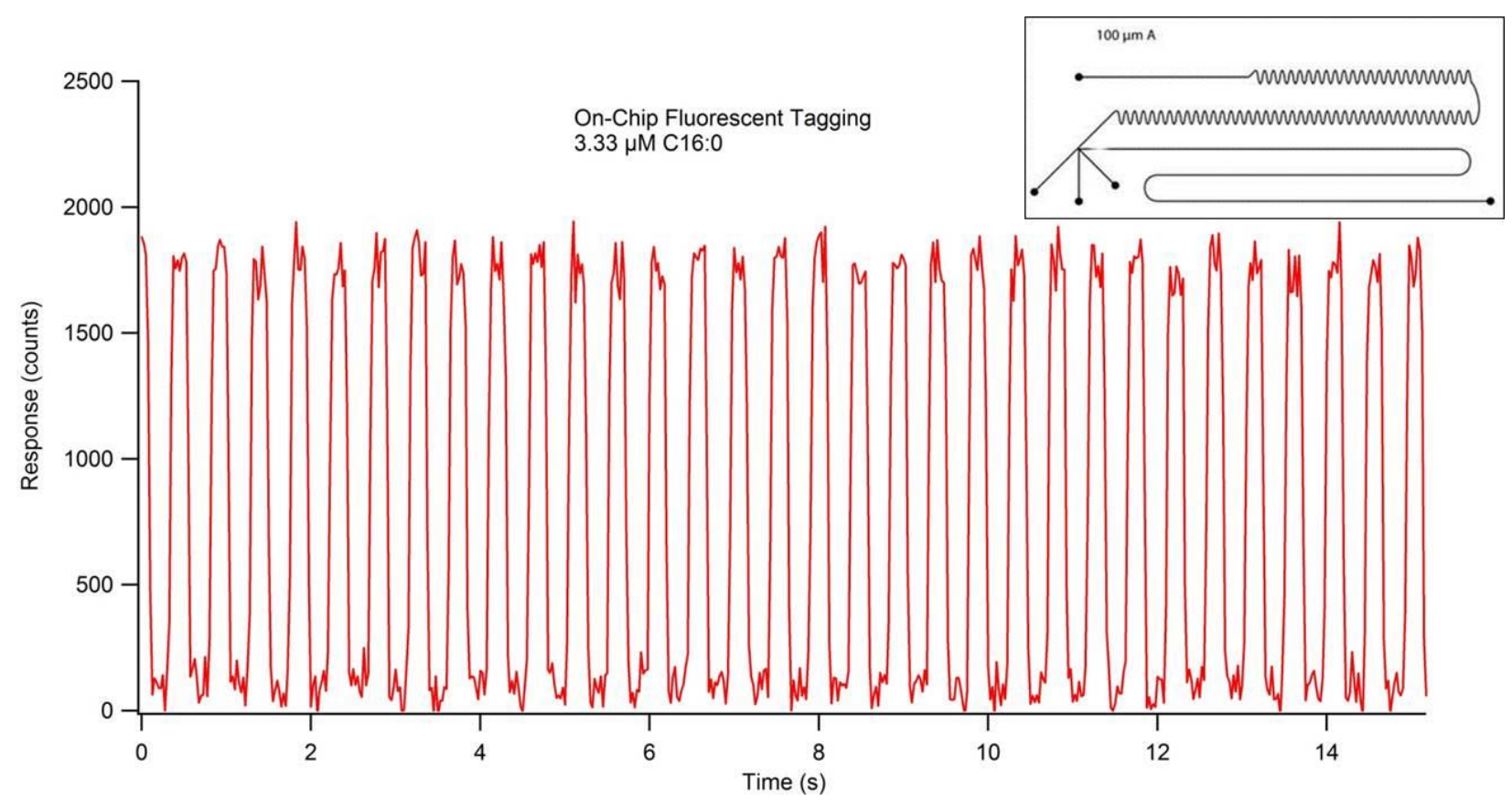

Figure 12. Fluorescence chronogram showing NDA-tagging reaction efficiency performed on-chip. Reactants were maintained in a 1:20:24 amine:KCN:NDA molar ratio and applied to the chip independently at $0.5 \mu \mathrm{L} / \mathrm{min}$. Using a chip design with 10 turns in the mixing region (inset) resulted in an incomplete reaction, indicative of inconsistent maximum peak responses.

Taken together, as shown in Figure 12 the detection limits of this microfluidic system was determined to be 436 amol per droplet. We estimate that under these conditions, we could 
accurately detect concentrations down to $10^{-17}$ moles per droplet, which provides an improvement of up to three orders of magnitude over that of the currently existing HPLC-MS methodology ( $20 \mathrm{fmol}$ LOD). As discussed in the next section, this can provide an accurate quantification system of sufficient sensitivity to complement other analytical platforms such as mass spectrometry.

\section{Discussion}

The PDMS microfluidic device fabrication process using photolithography has numerous benefits inclusive of low cost per chip ( $\$ 2$ or less), relative safety (HF required with glass chip fabrication process), rapid fabrication (approximately 1 day), and overall ease of use. The key drawback to using PDMS is the relatively low pressure thresholds that induce the chip to unbond from the glass surface. When the PDMS is oxidized by air plasma inside of the chamber, hydrocarbons are etched leaving behind surface silanol groups, resulting in a hydrophilic surface. When placed in contact with an oxidized glass surface, bridged siloxane bonds are formed at the interface, creating the seal. This seal, however, has the tendency to fail with increasing pressures applied to the chip from external syringe or HPLC pumps. The application of an epoxy coating at the perimeter of the chip and glass helps increase the pressure limits, however, there is still a tendency for relatively high pressures to force the fluid flowing through the channels to balloon outward, resulting in the chip failure and subsequent discarding.

During the photolithographic fabrication process, it is imperative to ensure a perfectly cleaned silicon wafer surface prior to spin coating. When conducting the soft bake process following UV exposure, the channel features will begin to faintly appear in the photoresist. This is caused by the exposed region underneath of the photomask to polymerize in the presence of UV light, resulting in this region being less soluble in the subsequent developing solution. Slight horizontal dispersion of the UV light through the photomask results in the channel width being slightly greater than that of the photomask. This is shown by the SEM micrograph having an average channel diameter of 155 $\mu \mathrm{m}$, as opposed to the $150 \mu \mathrm{m}$ width of the channels in the photomask.

The LIF detection system requires precise alignment of all optical components in order to achieve fluorescence detection, with minute adjustments required for optimal S/N. It was determined that a greater $\mathrm{S} / \mathrm{N}$ was achieved by complete removal of the rotation-mounted polarizer. This was somewhat expected when results showed the greatest response to be in a vertically polarized position, which is the native polarization of the incident laser. The increase in response by removing the polarizer is due to the percent transmittance of the polarizer being less than $100 \%$. In optimizing the pinhole filter diameter, the fluorescence response increased as the diameter of the pinhole filter was increased, up to $1.193 \mathrm{~mm}$, at which point no further response gain was observed. As the filter was opened past $1.193 \mathrm{~mm}$, a slight increase in noise was noticed while the signal remained constant. The goal of the pinhole filter is to block the lower, more variable outer regions of the laser beam. A cross-sectional view of the laser beam would show a Gaussian distribution in laser intensity, with the center of the laser having the greatest intensity and the outer regions showing higher variability. The dichroic lens displays an important function in that it acts as an additional filter, preventing the incident laser wavelength from reaching the detector. At a $425 \mathrm{~nm}$ longpass cutoff, the dichroic lens reflects all light with a wavelength less than $425 \mathrm{~nm}$ (incident laser wavelength $=405 \mathrm{~nm}$ ) and transmits all light with a wavelength greater than or equal to $425 \mathrm{~nm}$ (emission maxima $=470$ ). Of the four emission filters tested, the $472 \mathrm{~nm}$ bandpass filter provided the highest $\mathrm{S} / \mathrm{N}$ as it falls in line with the optimal emission maxima of NDA-tagged amines. A significant detector to detector response variability existed between the three detectors tested, with the Perkin Elmer APD displaying a significantly greater response as compared with the two EG\&G APDs. The positioning of the microchip on the sample stage is crucial to achieving a maximum $\mathrm{S} / \mathrm{N}$. To prevent misalignment of the chip, it is held in place using tape, and the entire stage is carefully adjusted in the $\mathrm{X}, \mathrm{Y}$, and Z planes. Optimal positioning of the focused laser beam should be in the center of the detection region of the channel, and the bottom of the glass should be within 1 
$\mathrm{mm}$ of the focusing microscope objective. Meticulous vertical alignment of the stage in relation to the microscope objective is required while monitoring real-time fluorescence response data until the greatest signal is achieved.

When optimizing flow rates for consistent droplet formation, it is necessary to have the immiscible oil phase be greater than that of the reactant(s) phase. The oil phase acts as a carrier fluid, with the reactants combining at the T-junction and tapering off into small volume droplets, which are carried down-channel by the immiscible oil. Due to the extremely low flow rates and relatively long length of tubing, a significant amount of time is usually required for stable flow to occur, resulting in consistent droplet formation. The on-chip NDA-tagging reactions shown in Figures 11 and 12 illustrate the importance of sufficient mixing. A linear channel exhibits laminar flow, with the only mixing occurring by simple inter-layer diffusion. By introducing the turns into the channel design, reorientation and folding of the fluidic layers within the individual droplets is induced. Each turn provides an additional fluidic layer interface, with subsequent turns rendering this fluidic layer infinitesimally thin to the point where simple diffusion becomes a fast and efficient form of mixing. Initial chip designs incorporating 10 turns into the mixing region proved insufficient, as shown by the resulting fluorescence chronogram. The varying peak widths found in Figure 11 are the result of unstable droplet formation, not that of incomplete reaction. The varying maximal response peak to peak shows that certain droplets exhibited greater reaction efficiency than others. Additionally, broad peaks (indicative of sample plugs rather than droplets) such as the one at approximately 2.75 minutes show a fluorescence response increasing within the individual droplet. This shows that the reaction efficiency at the front end of the droplet was worse than the efficiency at the tail end. Reaction efficiency was shown to be much improved using the modified chip design that incorporated 130 turns in the mixing region (Figure 12). The consistent maximal response for each droplet indicated that the reaction was complete.

The use of microfluidics with LIF was developed to provide the potential to detect ultra-trace quantities of bioactive lipids for use in lipidomics studies via fluorescence detection. This NDA chemical tagging schema may also be readily adapted for any amines and can have utility in metabolomics and proteomics studies. This is especially important in discovery studies, where typical quantification methodologies used in MS-based studies involving isotope enrichment are not feasible. To be a useful adjunct, the LIF system must have sensitivity that rivals or is of greater sensitivity than that of mass spectrometry platforms. Detection limits using this microfluidic device and LIF analysis system had detection capabilities at 436 amol per droplet. We estimate that under these conditions, we could accurately detect concentrations down to $10^{-17}$ moles per droplet, which provides an improvement of up to three orders of magnitude over that of the currently existing HPLC-MS methodology (20 fmol LOD). As LIF is a non-destructive detection technique, microfluidic device fabrication can be further modified to incorporate seamless integration with MS detection. Using this proposed analysis system design, LIF can be used for highly sensitive quantification, while parallel MS or other analytical methods can provide accurate identification of the tagged species. Of note, parallel complementary quantitation may be easily adapted for any amine, and may be useful in proteomics-based as well a lipidomics-based studies.

\section{.4. Materials and Methods}

Chemicals. Methanol was purchased from Fisher Scientific (Fair Lawn, NJ). Toluene, decylamine (C10:0), dodecylamine (C12:0), and octadecylamine (C18:0) were purchased from Sigma Aldrich (St. Louis, MO). Potassium cyanide (KCN), chlorotrimethylsilane, tetradecylamine (C14:0), hexadecylamine (C16:0), and oleylamine (C18:19) were purchased from Acros Organics (Bridgewater, NJ). 3-(2-furoyl)quinoline-2-carboxaldehyde (FQCA) and naphthalene dicarboxaldehyde (NDA) was purchased from Molecular Probes (Eugene, OR). Perfluorodecalin (PFD) was purchased from Alfa Aesar (Ward Hill, MA). SU-8 2015 positive epoxy photoresist and SU-8 developer were purchased from MicroChem (Newton, MA). Nano-Strip resist remover was 
purchased from Cyantek (Fremont, CA). Sylgard 184 silicone elastomer kit was purchased from Dow Corning (Midland, MI).

Instrumentation. Microchannel templates were designed using a vector graphics editor (Adobe Illustrator CC, Adobe Systems, Mountain View, CA) and chrome masks were fabricated via Front Range Photomask (Palmer Lake, CO). Uniform resist coating was applied to silicon wafers using a universal spin processor (WS-650 Spin Coater, Laurell Technologies, North Wales, PA) and photopolymerization of the resist was performed using a $500 \mathrm{~W}$ near-UV mercury arc lamp (Newport, Model 97435-1000-1, Newport Corporation, Irvine, CA). Hydrophilic rendering and oxidation of surfaces of finished PDMS microchips and glass bases were performed using air-plasma treatment (PCD-32G, Harrick Plasma, Ithaca, NY). Fluorescence excitation was performed using a variable power $50 \mathrm{~mW} 405 \mathrm{~nm}$ blue laser (DL-405-050, CrystaLaser, Reno, NV) and emission detected using a single photon counting module (PerkinElmer SPCM-AQR-14, Excelitas Technologies, Waltham, MA). All optical filters and components were purchased from Omega Optical (Brattleboro, VT), Edmund Optics (Barrington, NJ), and ThorLabs (Newton, NJ). Pre-chip chromatographic separations were performed using a high performance liquid chromatograph (Ultimate 3000, Dionex Corporation, Sunnyvale, CA) and microbore C 18 column (XTerra, 5 $\mathrm{mm}, 0.32 \times$ $50 \mathrm{~mm}$, Waters Corporation, Pittsburgh, PA). All data was collected and processed using graphical programming software (LabView 2012, National Instruments, Austin, TX).

Fluorescent Labeling of Amines. For optimal fluorescent tagging of amines using FQCA, $10 \mathrm{mM}$ solutions of both FQCA and amine were prepared in methanol, and a $10 \mathrm{mM}$ solution of KCN was prepared in water. Optimal reaction conditions were determined to be 1:10:12 (v/v/v) equimolar amine:KCN:FQCA. The reactants were mixed in an amber glass vial and allowed to react for approximately 45 minutes at $40^{\circ} \mathrm{C}$ using a heating block. Fluorescent tagging of amines using NDA follows a similar procedure to that of FQCA. Each reactant stock solution was prepared at $10 \mathrm{mM}$ with both NDA and amine in methanol and $\mathrm{KCN}$ in water. The reactants were mixed in an amber glass vial and heated at $40^{\circ} \mathrm{C}$ for approximately 30 minutes for the reaction to proceed to completion.

Reversed Phase HPLC Separation of Primary Fatty Acid Amines. A Waters 1525 Binary HPLC Pump with Waters 2475 Multi Wavelength Fluorescence Detector and Waters Breeze version 3.30 software package was used for separation and fluorescence detection of amine mixtures. A Waters Xterra MS C18 with 5 um particle size, $3.9 \mathrm{~mm} \times 150 \mathrm{~mm}$ column was used to separate saturated fluorescently tagged amine standards. A $5 \mathrm{uL}$ injection loop was used for consistent injection volumes and the column was maintained at room temperature. An isocratic elution profile consisting of $100 \%$ methanol with a flow rate of $1.5 \mathrm{~mL} / \mathrm{min}$ was used. For analysis of FQCA-tagged amines, an excitation wavelength of $488 \mathrm{~nm}$ and an emission wavelength of $590 \mathrm{~nm}$ was used with gain and energy units full scale (EUFS) settings at 100 and 1,000, respectively. For analysis of NDA-tagged amines, an excitation wavelength of $405 \mathrm{~nm}$ and emission wavelength of $470 \mathrm{~nm}$ was used with gain and EUFS settings at 100 and 1,000, respectively.

Microchip Fabrication. Microchannel designs were patterned onto chrome coated soda lime glass at a resolution of $\pm 0.5 \mu \mathrm{m}$ to yield a 6 by 6 inch circular photomask. Approximately $5 \mathrm{~mL}$ of SU-8 2015 photoresist was applied to the center of a $150 \mathrm{~mm}$ silicon wafer and spin coated to a uniform thickness of approximately $108 \mu \mathrm{m}$. A spin coater was programmed to ramp to $500 \mathrm{rpm}$ for 10 seconds followed by a second ramp to 2,000 rpm for 30 seconds. The resist-coated wafer was then soft-baked at $65^{\circ} \mathrm{C}$ for $5 \mathrm{~min}$, returned to room temperature, and then heated to $95^{\circ} \mathrm{C}$ for $25 \mathrm{~min}$. UV exposure of the resist-coated wafer through the photomask was performed for 30 seconds at a distance of $20 \mathrm{~cm}$ and resulted in visible channel designs appearing in the resist. The wafer underwent a post-exposure bake at $65^{\circ} \mathrm{C}$ for 5 minutes, returned to room temperature, and was again heated to $95^{\circ} \mathrm{C}$ for 12 minutes. The wafer was developed for $10 \mathrm{~min}$ to remove all un-exposed resist, and was rinsed with additional fresh developer, isopropyl alcohol, and deionized water and dried under a stream of nitrogen gas. At this point, an optional hard bake curing could 
be performed at $200{ }^{\circ} \mathrm{C}$ for 15 minutes, however, this was deemed unnecessary as the positive resist channel designs were firmly deposited on the wafer.

The PDMS was prepared by mixing the silicon elastomer base and curing agent in a 10:1 (m/m), respectively. The mixture was then desiccated to remove all air bubbles that formed during mixing. The PDMS was carefully poured over the master to ensure that there were no air bubbles on the surface and cured for at least $1 \mathrm{~h}$ at $100{ }^{\circ} \mathrm{C}$. After curing, the PDMS polymer was gently peeled from the master and inlet ports were punched at their designated locations. After being thoroughly cleaned using isopropyl alcohol and dried, the PDMS microchip and glass base were plasma cleaned for 3-5 min at high power. Once removed, the substrates were applied to one another to form a spontaneous, irreversible seal. To ensure the seal under pressure driven flow, quick-setting epoxy was applied to the perimeter of the PDMS chip and glass base. 1/32" OD PEEK tubing was inserted into each port and epoxy was used to provide a leak-proof seal.

Scanning Electron Microscopy. A Hitachi S-3400N scanning electron microscope (SEM) was used for analysis and verification of microfluidic device features. Prior to bonding to glass, a clean microfluidic chip was positioned feature side up on a $15 \mathrm{~mm}$ aluminum sample stub affixed to a copper stub holder. The high voltage was turned off and the SEM chamber was exposed to air prior to opening. Once opened, the stub holder, stub, and sample were placed onto the specimen stage and the total height was measured to ensure no contact is made with the metal optics guard. This height was inputted into the software program and the stage height was adjusted accordingly (sample should be approximately $1 \mathrm{~mm}$ from the optics guard). The chamber was closed and air was evacuated with a vacuum pump until internal pressure reached $50 \mathrm{~Pa}$. With an accelerating voltage of $15.0 \mathrm{kV}$, the sample image brought into focus by adjusting magnification and focus knobs on the external controller. Brightness and contrast settings were also adjusted to provide the sharpest image. Both two- and three-dimensional images of the microfluidic device were acquired.

Development of a Laser Induced Fluorescence Detection System. For the development of a LIF detection system, all optical components were purchased from ThorLabs. The excitation source was a CrystaLaser Violet Blue solid-state laser with a wavelength of $405 \mathrm{~nm}( \pm 5 \mathrm{~nm})$, variable power source from 0-50 $\mathrm{mW}$, and vertical polarization. The laser beam is reflected off of a series of mirrors and passes through a $405 \mathrm{~nm}$ bandpass excitation filter, a rotation-mounted linear polarizer set to $300^{\circ}$, and a pinhole filter. A longpass dichroic lens with cutoff wavelength of $425 \mathrm{~nm}$ reflected incident laser light vertically through a 40x microscopic objective lens, focusing the beam to the desired detection region of the microfluidic device. The resulting fluorescence emission in the downward vertical plane passed back through the microscope objective and dichroic lens to a final mirror to reflect the fluorescence light horizontally to an optics tube. The optics tube contains a pinhole filter at the front end with a $472 \mathrm{~nm}$ emission bandpass filter and achromatic focusing lens within. The fluorescence emission beam is focused onto the $180 \mu \mathrm{m}$ diameter circular active area of a single photon counting module equipped with a silicon avalanche photodiode array detector (APD). Individual photons generate transistor-transistor logic pulses, which are binned over $25 \mathrm{~ms}$ increments and transmitted to LabView data acquisition software.

Preliminary LIF detection system alignment was conducted by visually aligning the excitation laser beam downstream from component to component. Each optical component has alignment knobs to adjust both the horizontal and vertical angles of the component. In lieu of a fluorescing sample being placed on the horizontal sample stage above the focusing microscope objective, a mirror was used to reflect the laser beam back to the center of the dichroic lens. In order to align the optical components downstream from the second passing of the dichroic lens, a concentrated NDA-tagged dodecylamine solution was loaded into a capped, flat-sided quartz cuvette and placed on the sample stage. The resonant fluorescence beam was aligned through the dichroic lens, reflected by a mirror, and through the optics tube. A small, handheld dental mirror was used to ensure the fluorescence beam was properly focused from the optics tube onto the active area of the 
photon counting detector. Finally, a microfluidic chip undergoing segmented flow with NDA-tagged dodecylamine was placed on the sample stage and the stage aligned until the excitation laser beam was focused directly within a sample channel. Optical components downstream from the sample stage as well as the sample stage horizontal and vertical alignment were adjusted until the detected fluorescence droplet response achieved the greatest $S / N$. All alignment procedures are completed in complete darkness to allow for maximum excitation and emission beam visibility.

Several optical components required optimization to achieve the greatest possible $\mathrm{S} / \mathrm{N}$ of NDA-tagged amine. Signal was recorded while adjusting a pinhole filter from closed to its maximum diameter of approximately $2.6 \mathrm{~mm}$, and precise diameters were measured using a caliper. The laser polarization was adjusted from vertical to horizontal alignment, as well as with the rotation-mounted polarizer removed from the beam path. Several emission filters inclusive of 495 $\mathrm{nm}$ and $472 \mathrm{~nm}$ bandpass filters and $470 \mathrm{~nm}$ and $450 \mathrm{~nm}$ longpass filters were tested. Using the variable power controller, the lasing power was tested between $5 \mathrm{~mW}$ and $50 \mathrm{~mW}$. To determine variance caused by specific APDs, a Perkin Elmer APD was tested against two different models of EG\&G APDs. On-chip fluorescent tagging efficiency was examined using two different channel designs; one design contained a droplet mixing region containing 10 turns before the linear detection channel and a second design contained 130 turns before the detection channel. A preliminary investigation of pre-chip chromatographic separation was conducted using a Dionex Ultimate 3000 HPLC Pump with a Waters Xterra MS C18 column $(5 \mu \mathrm{m}, 3.9 \mathrm{~mm} \times 150 \mathrm{~mm})$. The flow rate was held constant at $4 \mu \mathrm{L} / \mathrm{min}$ and a $2 \mu \mathrm{L}$ injection volume was used.

Droplet Based Microfluidic Flow. The formation of droplet-based flow within the microfluidic device is crucial for conducting on-chip reactions. To optimize droplet formation flow rates, only two inlet ports were used. The inlet port with the channel that continues straight past the T-junction was used for the flow of perfluorodecalin, an immiscible oil-phase. One of the three inlet ports with channels entering the T-junction (perpendicular to the oil-phase channel) was used for the flow of NDA-tagged dodecylamine. Perfluorodecalin and the tagged-amine solution were loaded into separate syringes and applied to the microchip using independent syringe pumps. Flow rates were adjusted until rapid and consistent droplets were formed on-chip. Droplet formation was detected visually due to differences in light refraction properties of perfluorodecalin and methanol, as well as by fluorescence detection.

To conduct on-chip NDA-tagging reactions, a microchip was used with all four inlet ports accessible with PEEK tubing. The oil-phase inlet was maintained as previously described, with the other three inlets used for the three reactants in the NDA-tagging reaction. Amine, KCN, and NDA solutions were prepared at a concentration ratio of 1:20:24, respectively, and each was loaded into a syringe. The three reactant syringes were loaded onto a multi-syringe pump and all applied to the microchip simultaneously at the same flow rate. Flow rates were optimized for rapid and consistent droplet formation.

\section{Conclusions}

The ability to conduct fluorescent tagging reactions and the incorporation of chromatographic separations on-chip enables the possibility of a true $\mu$ TAS system of analysis, dramatically reducing any errors caused by sample handling. Microfluidics also presents the opportunity to move towards a green analysis system, with a dramatic reduction of chemical and sample usage due to the extremely low flow rates and volumes on-chip. Further work towards optimizing on-chip chromatographic separation efficiency by addressing the inherent PDMS robustness issues will be required prior to the investigation of using a microfluidic system for the analysis and highly sensitive detection of biologically endogenous PFAMs. 
Author Contributions: A.D. and M.C. designed research; A.D. conducted all studies and data analyses: MC oversaw all studies: A.D. and M.C. wrote and reviewed the manuscript. All authors have read and agreed to the published version of the manuscript.

Funding: This research was funded by the National Institute of Health, grant number R15 NS038443

Acknowledgments: The authors thank the late Dr. Mitchell E. Johnson for support and inspiration of this work.

Conflicts of Interest: The authors declare no conflict of interest

\section{References}

1. Divito, E.B. and M. Cascio, Metabolism, physiology, and analyses of primary fatty acid amides. Chem Rev, 2013. 113(10): p. 7343-53.

2. Arafat, E.S., et al., Identification of fatty acid amides in human plasma. Life Sci, 1989. 45(18): p. $1679-87$.

3. Cravatt, B.F., et al., Chemical characterization of a family of brain lipids that induce sleep. Science, 1995. 268(5216): p. 1506-9.

4. Lerner, R.A., et al., Cerebrodiene: a brain lipid isolated from sleep-deprived cats. Proc Natl Acad Sci U S A, 1994. 91(20): p. 9505-8.

5. Cravatt, B.F., R.A. Lerner, and D.L. Boger, Structure Determination of an Endogenous Sleep-Inducing Lipid, cis-9-Octadecanamide (Oleamide): A Synthetic Approach to the Chemical Analysis of Trace Quantities of a Natural Product. J Am Chem Soc, 1996. 118(3): p. 580-90.

6. Hoi, P.M. and C.R. Hiley, Vasorelaxant effects of oleamide in rat small mesenteric artery indicate action at a novel cannabinoid receptor. Br J Pharmacol, 2006. 147(5): p. 560-8.

7. Sudhahar, V., S. Shaw, and J.D. Imig, Mechanisms involved in oleamide-induced vasorelaxation in rat mesenteric resistance arteries. Eur J Pharmacol, 2009. 607(1-3): p. 143-50.

8. Huitron-Resendiz, S., et al., Effect of oleamide on sleep and its relationship to blood pressure, body temperature, and locomotor activity in rats. Exp Neurol, 2001. 172(1): p. 235-43.

9. Martinez-Gonzalez, D., et al., Oleamide and anandamide effects on food intake and sexual behavior of rats. Neurosci Lett, 2004. 364(1): p. 1-6.

10. Fedorova, I., et al., Behavioral evidence for the interaction of oleamide with multiple neurotransmitter systems. J Pharmacol Exp Ther, 2001. 299(1): p. 332-42.

11. Huidobro-Toro, J.P. and R.A. Harris, Brain lipids that induce sleep are novel modulators of 5-hydroxytrypamine receptors. Proc Natl Acad Sci U S A, 1996. 93(15): p. 8078-82.

12. Huidobro-Toro, J.P., C.F. Valenzuela, and R.A. Harris, Modulation of GABAA receptor function by G protein-coupled 5-HT2C receptors. Neuropharmacology, 1996. 35(9-10): p. 1355-63.

13. Boger, D.L., J.E. Patterson, and Q. Jin, Structural requirements for 5-HT2A and 5-HT1A serotonin receptor potentiation by the biologically active lipid oleamide. Proc Natl Acad Sci U S A, 1998. 95(8): p. 4102-7.

14. Thomas, E.A., et al., Unique allosteric regulation of 5-hydroxytryptamine receptor-mediated signal transduction by oleamide. Proc Natl Acad Sci U S A, 1997. 94(25): p. 14115-9.

15. Boger, D.L., et al., Chemical requirements for inhibition of gap junction communication by the biologically active lipid oleamide. Proc Natl Acad Sci U S A, 1998. 95(9): p. 4810-5. 
16. Guan, X., et al., The sleep-inducing lipid oleamide deconvolutes gap junction communication and calcium wave transmission in glial cells. J Cell Biol, 1997. 139(7): p. 1785-92.

17. Hamberger, A. and G. Stenhagen, Erucamide as a modulator of water balance: new function of a fatty acid amide. Neurochem Res, 2003. 28(2): p. 177-85.

18. Mitchell, C.A., et al., Enhancement of neovascularization in regenerating skeletal muscle by the sustained release of erucamide from a polymer matrix. J Biomater Appl, 1996. 10(3): p. 230-49.

19. Wakamatsu, K., et al., Isolation of fatty acid amide as an angiogenic principle from bovine mesentery. Biochem Biophys Res Commun, 1990. 168(2): p. 423-9.

20. Huang, J.K. and C.R. Jan, Linoleamide, a brain lipid that induces sleep, increases cytosolic Ca2+ levels in MDCK renal tubular cells. Life Sci, 2001. 68(9): p. 997-1004.

21. Wang, G., et al., Long-chain ceramide is elevated in presenilin 1 (PS1M146V) mouse brain and induces apoptosis in PS1 astrocytes. Glia, 2008. 56(4): p. 449-56.

22. Clapham, D.E., Calcium signaling. Cell, 1995. 80(2): p. 259-68.

23. Devane, W.A., et al., Isolation and structure of a brain constituent that binds to the cannabinoid receptor. Science, 1992. 258(5090): p. 1946-9.

24. Nichols, K.K., et al., Identification of fatty acids and fatty acid amides in human meibomian gland secretions. Invest Ophthalmol Vis Sci, 2007. 48(1): p. 34-9.

25. Di Marzo, V. and A. Fontana, Anandamide, an endogenous cannabinomimetic eicosanoid: 'killing two birds with one stone'. Prostaglandins Leukot Essent Fatty Acids, 1995. 53(1): p. 1-11.

26. Bisogno, T., et al., The sleep inducing factor oleamide is produced by mouse neuroblastoma cells. Biochem Biophys Res Commun, 1997. 239(2): p. 473-9.

27. Sultana, T. and M.E. Johnson, Sample preparation and gas chromatography of primary fatty acid amides. J Chromatogr A, 2006. 1101(1-2): p. 278-85.

28. Hanus, L.O., et al., A gas chromatographic-mass spectral assay for the quantitative determination of oleamide in biological fluids. Anal Biochem, 1999. 270(1): p. 159-66.

29. Brautigam, A., et al., Rapid and simple UPLC-MS/MS method for precise phytochelatin quantification in alga extracts. Anal Bioanal Chem, 2010. 398(2): p. 877-83.

30. Neususs, C., M. Pelzing, and M. Macht, A robust approach for the analysis of peptides in the low femtomole range by capillary electrophoresis-tandem mass spectrometry. Electrophoresis, 2002. 23(18): p. 3149-59.

31. Divito, E.B., et al., Electrospray ionization and collision induced dissociation mass spectrometry of primary fatty acid amides. Anal Chem, 2012. 84(5): p. 2388-94.

32. Gee, A.J., L.A. Groen, and M.E. Johnson, Ion trap mass spectrometry of trimethylsilylamides following gas chromatography. J Mass Spectrom, 2000. 35(3): p. 305-10.

33. Carpenter, T., et al., Use of reversed phase HP liquid chromatography to assay conversion of N-acylglycines to primary fatty acid amides by peptidylglycine-alpha-amidating monooxygenase. J Chromatogr B Analyt Technol Biomed Life Sci, 2004. 809(1): p. 15-21.

34. Feng, L. and M.E. Johnson, Selective fluorescence derivatization and capillary electrophoretic separation of amidated amino acids. J Chromatogr A, 1999. 832: p. 211-224. 
35. Whitesides, G.M., The origins and the future of microfluidics. Nature, 2006. 442(7101): p. 368-73.

36. Duffy, D.C., et al., Rapid Prototyping of Microfluidic Systems in Poly(dimethylsiloxane). Anal Chem, 1998. 70(23): p. 4974-84.

37. Song, H., D.L. Chen, and R.F. Ismagilov, Reactions in droplets in microfluidic channels. Angew Chem Int Ed Engl, 2006. 45(44): p. 7336-56.

38. Stroock, A.D., et al., Chaotic mixer for microchannels. Science, 2002. 295(5555): p. 647-51.

39. Aref, H., Stirring by chaotic advection. J. Fluid Mech., 1984. 143: p. 1-21.

40. Song, H., et al., Experimental test of scaling of mixing by chaotic advection in droplets moving through microfluidic channels. Appl Phys Lett, 2003. 83(12): p. 4664-4666.

41. Ottino, J.M., Mixing and chemical reactions: a tutorial. Chem Eng Sci, 1994. 49: p. 4005-4027.

42. Beebe, D.J., G.A. Mensing, and G.M. Walker, Physics and applications of microfluidics in biology. Annu Rev Biomed Eng, 2002. 4: p. 261-86. 